\section{Massive pleural effusion due to pancreatic pseudocyst}

\author{
A R J Girbes, P E Postmus, W Jansen, \\ E J vd Jagt, J H Kleibeuker
}

\begin{abstract}
Four patients with massive pleural effusions due to pancreatic pseudocysts who were initially treated conservatively with percutaneous pleural drainage are described. In two patients the pleural effusions and the pancreatic pseudocysts disappeared, but two required surgery, in one case because conservative treatment failed and in the other at a later date for a recurrent pancreatic pseudocyst.
\end{abstract}

Massive pleural effusion is a well known but rare complication of pancreatic pseudocyst. ${ }^{1-4}$ It is due to a direct communication between either a subdiaphragmatic or a mediastinal pseudocyst and the pleural cavity. Massive pleural effusion may be the presentation of pancreatic pseudocyst and may lead to diagnostic difficulties if not considered. We describe four patients who were all initially treated conservatively.

\section{Case reports}

CASE 1

A 48 year old man was referred because of progressive shortness of breath on exertion. He had previously experienced multiple blunt abdominal trauma not requiring medical treatment and until six months before admission had consumed 10-15 units of alcohol daily. He had lost $8 \mathrm{~kg}$ in weight during the preceding six months. Examination revealed signs of a pleural effusion at both lung bases, to a greater extent on the left side. There was no peripheral oedema, ascites, or hepatosplenomegaly.

Serum amylase activity was raised (table). A chest radiograph showed a massive pleural effusion on the left and a small effusion on the right. Aspiration on the left side yielded yellowish fluid with a raised amylase content (table), a few leucocytes, and no malignant cells. Ultrasonography of the abdomen showed cystic changes of the pancreatic head without ascites. Computed tomography showed a pancreatic pseudocyst.

Communication between a pancreatic pseudocyst and the pleural cavities was diagnosed. Percutaneous pleural drainage with continuous suction on the left side resulted in a decrease of pleural fluid on both sides. After four weeks, with minimal fluid production on the left, the amount of fluid on the right increased rapidly. Aspiration again disclosed a high amylase content and a second drain was introduced. Further treatment consisted of monomeric nutrition through a nasojejunal tube. After two months the pleural drainage was terminated and there was no recurrence of the pleural effusions. Serum amylase activity and albumin concentration returned to normal. Computed tomography showed disappearance of the pancreatic pseudocyst. The patient was discharged in good physical health and 18 months later has had no recurrence of the pleural effusions.

\section{CASE 2}

A 33 year old man was referred with recurrent pancreatitis six months after being admitted for pancreatitis due to chronic alcohol abuse. He complained of intermittent epigastric pain and had raised serum amylase activity (table). His chest radiograph was normal. He was treated with nasogastric suction and parenteral nutrition. The abdominal pain and the serum amylase both decreased. Two months later he developed progressive shortness of breath on exertion. A left sided massive pleural effusion was found. Aspiration disclosed an exudate with a raised amylase content (table). We concluded that a pancreatic pseudocyst had perforated into the pleural cavity and he was treated with repeated pleuracenteses. After six weeks the pleural effusion had resolved, serum amylase activity was normal, and the patient was discharged in good health. Although he continued to drink and had many more episodes of pancreatitis, requiring surgery on four occasions, he had had no recurrence of the pleural effusion 13 years later.

Results of laboratory investigations on paired pleural fluid and serum specimens

\begin{tabular}{|c|c|c|c|c|c|c|c|c|c|c|c|c|}
\hline \multirow[b]{2}{*}{ Patient No } & \multicolumn{4}{|l|}{ Fluid } & \multicolumn{4}{|l|}{ Serum } & \multicolumn{4}{|c|}{ Fluid:serum ratio } \\
\hline & 1 & 2 & 3 & 4 & 1 & 2 & 3 & 4 & 1 & 2 & 3 & 4 \\
\hline Amylase $\left(\mathrm{U} / 1, \times 10^{3}\right)$ & $63 \cdot 1$ & $16 \cdot 4$ & $180 \cdot 0$ & $3 \cdot 3$ & 1.6 & $5 \cdot 2$ & 1.7 & $1 \cdot 3$ & $39 \cdot 5$ & $3 \cdot 2$ & 104 & $2 \cdot 6$ \\
\hline Proteins $(\mathbf{g} / \mathbf{l})$ & 23 & 29 & 39 & 41 & 54 & 62 & 55 & 66 & 0.4 & 0.5 & 0.7 & 0.6 \\
\hline $\mathrm{LDH}(\mathrm{U} / \mathrm{l})$ & 393 & 103 & 206 & ND & 187 & 147 & 190 & 120 & $2 \cdot 1$ & 0.7 & $1 \cdot 1$ & ND \\
\hline Glucose $(\mathrm{mmol} / \mathrm{l})$ & $4 \cdot 0$ & 5.0 & 6.9 & ND & 3.8 & 5.4 & $5 \cdot 7$ & 6.0 & $1 \cdot 1$ & 0.9 & 1.0 & ND \\
\hline
\end{tabular}

ND-not done. 
CASE 3

A 35 year old man developed progressive shortness of breath two years after receiving conservative treatment for pancreatitis due to alcohol abuse. Physical examination and a chest radiograph showed a large right sided pleural effusion. Serum amylase activity was increased (table). An exudate with a raised amylase content (table) was aspirated. Ultrasonography of the abdomen showed cystic changes in the pancreas without ascites and computed tomography confirmed the presence of a pancreatic pseudocyst. A communication between a pancreatic pseudocyst and the pleural cavity was diagnosed. Percutaneous drainage on the right side with continuous suction was continued for four weeks. Further treatment included nasojejunal tube feeding. Soon after chest tube suction was stopped the pleural effusion reappeared, so the cyst was drained surgically. Two months later further surgery was performed because of recurrence of the pancreatic pseudocyst. One year later there had been no further pancreatitis or pleural effusions.

CASE 4

A 51 year old man was admitted because of continuous abdominal pain. One year earlier he had had acute pancreatitis and laparotomy showed only an enlarged pancreas. Alcohol use was denied. On admission a mass was felt in the left upper abdomen. Serum amylase activity was substantially raised (table). A chest radiograph was normal. Several days after admission the patient suddenly experienced abdominal pain and shortness of breath. The abdominal mass had disappeared and a left sided massive pleural effusion was found. Aspiration disclosed an exudate with increased amylase activity (table). A chest tube was inserted and continuous suction was started. After four weeks this was terminated and the pleural effusion did not recur. The patient is well 16 years later.

\section{Discussion}

Massive pleural effusion complicating a pancreatic pseudocyst is rare. The history of patients with this complication often does not suggest chronic pancreatic disease. ${ }^{13}$ Such patients usually complain of dyspnoea. In only 14 of 34 patients with internal pancreatic fistulas was there a history of concomitant acute pancreatitis ${ }^{1}$ and in seven patients with pancreatic pleural effusion only two had abdominal complaints. ${ }^{4}$ Two of our patients presented with dyspnoea without a history of concomitant pancreatitis. In the two other patients massive pleural effusions developed acutely during conservative treatment of their pancreatitis. Pancreatic pseudocyst may complicate pancreatitis of any cause, but most result from alcoholic pancreatitis $(40-80 \%)$ and internal pancreatic fistula in chronic pancreatitis is especially associated with alcohol consumption $(82 \%){ }^{13}$

Treatment can be either conservative or operative drainage. Drainage by chest tube or repeated thoracocenteses is usually effective in pancreatic pleural effusions ${ }^{13}$ but sometimes early surgery is preferred. ${ }^{1}$ Another approach is percutaneous drainage of the abdominal pancreatic pseudocyst, guided by computed tomography, when pleural drainage fails. ${ }^{5}$ Our patients were initially treated conservatively with drainage of the pleural effusion and complementary measures for the existing pancreatitis (nasogastric suction, nasojejunal tube feeding, or parenteral feeding). In two patients (Nos 1 and 4) the pleural effusions as well as the pancreatic pseudocyst disappeared and there were no sequelae. These patients also stopped their alcohol abuse.

In one patient (No 2) the pleural effusions disappeared completely, but later abdominal surgery was necessary because of recurrence of the pancreatic pseudocyst during continued alcohol abuse. In one patient (No 3) conservative treatment was not successful and this patient was operated on because of the rapid recurrence of pleural effusions. These different outcomes indicate the problem of predicting the clinical course and planning optimal treatment in advance. On the basis of our experience and of published reports, we believe that primary conservative treatment is justified and that thoracic drainage should be continued for several weeks if required.

1 Cameron JL, Kieffer RS, Anderson WJ, Zuidema GD. Internal pancreatic fistulas: pancreatic ascites and pleural effusions. Ann Surg 1976;184:587-93.

2 Anderson WJ, Skinner DB, Zuidema GD, Cameron JL. Chronic pancreatic pleural effusions. Surg Gynaecol Obstet 1973;137:827-30.

3 Kane MG, Krejs GJ. Pancreatic pseudocyst. Adv Intern Med 1984;29:271-300.

4 Cameron JL. Chronic pancreatic ascites and pancreatic pleural effusions. Gastroenterology 1978;74:134-40.

5 Gerzog SG, Johnson WC, Robbins AH, Spechler SJ, Nabseth DC. Percutaneous drainage of infected pancreatic pseudocyst. Arch Surg 1984;119:888-93. 